\title{
IUCF HIGH INTENSITY POLARIZED ION SOURCE
}

\author{
M. Wedekind, R. Brown, V. Derenchuk, T. Ellison, D. Friesel, J. Hicks, \\ D. Jenner, A. Pei, H. Petri, P. Schwandt and J. Sowinski \\ Indiana University Cyclotron Facility, Bloomington, IN 47408
}

\section{INTRODUCTION}

We have completed approximately nine person-years of work on the high intensity polarized ion source (HIPIOS), or $45 \%$ of the total project. Due to manpower and operating budget limitations, we determined last fall that continuing with the ANAC ECR installation was not compatible with finishing HIPIOS, the high voltage terminal (Terminal C) and the injection beam line (BL1C) on a reasonable schedule. Consequently, in October we redirected our project efforts toward HIPIOS. We are allowing the schedule for the Terminal $\mathrm{C}$ and BL1C projects to slip along with construction of the new building addition in order to concentrate on completing HIPIOS testing. The NSF has awarded IUCF approximately $\$ 1 \mathrm{M}$ of the $\$ 1.3 \mathrm{M}$ HIPIOS/BL1C grant. The third and final disbursement is expected next month.

\section{ATOMIC BEAM SECTION ASSEMBLY AND TESTING}

We have started assembling the source (Fig. 1) in the south end of the Cooler building for off-line testing where it will remain until Terminal $\mathrm{C}$ is installed in the new building addition. Assembly of the dissociator vacuum chamber and vacuum system is complete. As of this writing, atomic beam velocity measurements are in progress. Over the summer we will perform a series of tests to look at the flux density of the cold atomic beam without the sextupoles, with the sextupoles in place but not energized and with the sextupoles energized. We will then run the same tests with the RF transition units in place.

\section{A. Dissociator}

Measurements of the atomic beam velocity without sextupoles have been made using an atomic beam chopper generously loaned to IUCF by Professor W. Grüebler of ETH. Results indicate that with an $\mathrm{H}_{2}$ flow rate of about $20 \mathrm{SCCM}$, and a nozzle temperature of $25^{\circ} \mathrm{K}, 30^{\circ} \mathrm{K}$ and $40^{\circ} \mathrm{K}$, the atomic beam velocity was consistent with a beam temperature of $35^{\circ} \mathrm{K}, 42^{\circ} \mathrm{K}$ and $54^{\circ} \mathrm{K}$ respectively (Fig. 2).

There continues to be a build up of white powder in the cold nozzle and a milky film formed on the dissociator tube during operation. Replacing the original pyrex tube with a quartz one has not alleviated the problem. We are currently installing an $\mathrm{LN}_{2}$ zeolite trap on the hydrogen gas line to remove impurities.

\section{B. Sextupoles}

Both sextupoles have been mapped and are ready for installation. We increased the length of the first sextupole by $50 \%$ (from $10 \mathrm{~cm}$ to $15 \mathrm{~cm}$ ) from previous designs to provide the increased integrated sextupole strength recommended by TUNL. The ray 


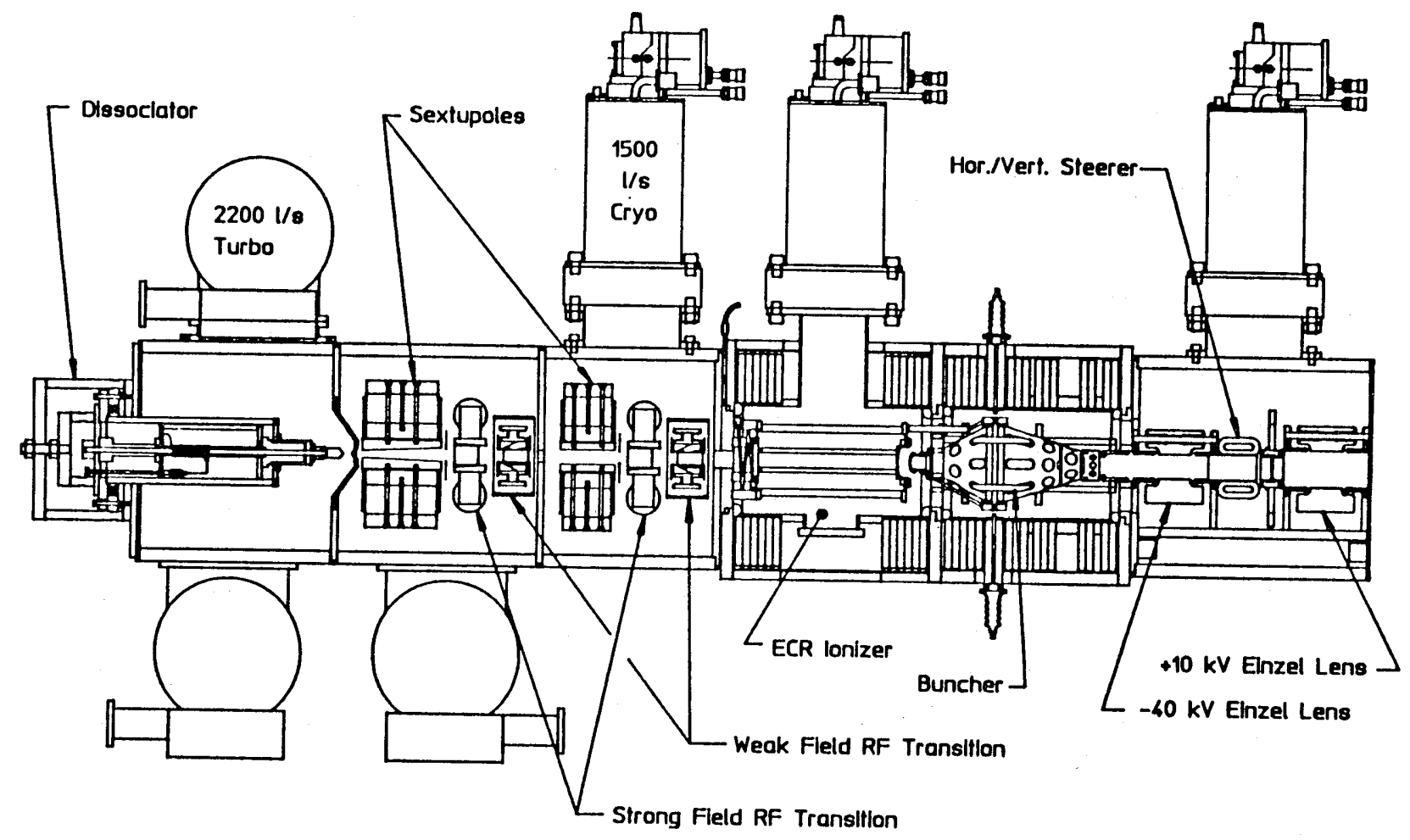

$\stackrel{\mathrm{Ft} . \longrightarrow}{\longrightarrow}$

HIGH INTENSITY POLARIZED ION SOURCE

Figure 1. High Intensity Polarized Ion Source

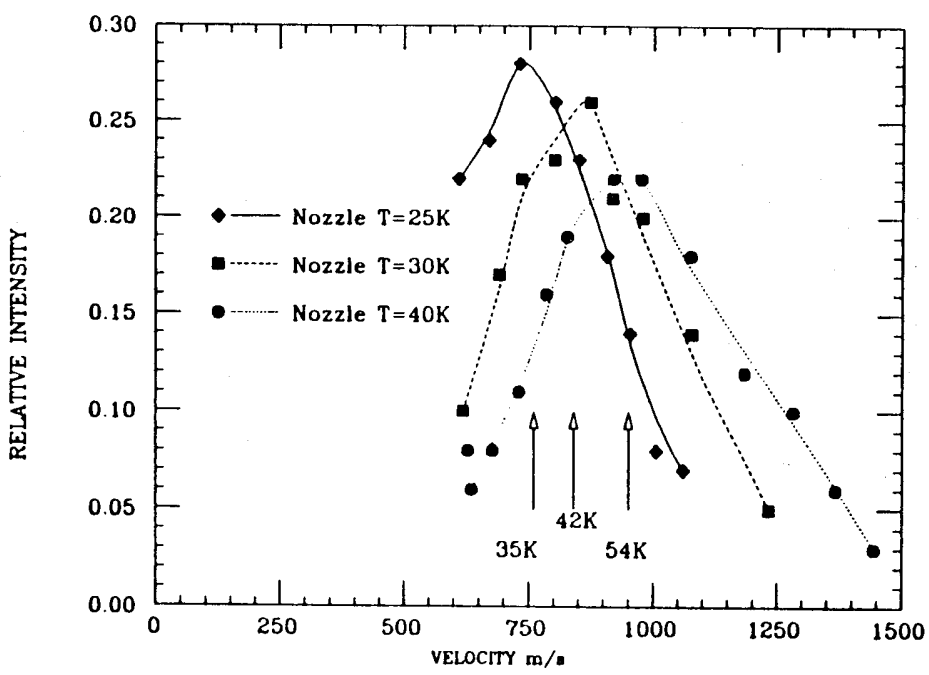

Figure 2. Atomic beam velocity for Hydrogen measured with nozzle temperatures of $25^{\circ} \mathrm{K}$, $30^{\circ} \mathrm{K}$, and $40^{\circ} \mathrm{K}$. Relative intensities between curves is arbitrary. 
trace code predicts an optimum integrated field of $105 \mathrm{kG}-\mathrm{cm}$ for the first sextupole; the actual integrated field is $112.7 \mathrm{kG}-\mathrm{cm}$. We have measured a maximum pole tip field for the first sextupole of $7600 \mathrm{G}$ at the design current of $250 \mathrm{Amps}$, and $5600 \mathrm{G}$ for the second sextupole at the design current of 200 Amps.

\section{Rf Transitions}

The rf transitions, which convert the atomic polarization to nuclear polarization, utilize the conventional adiabatic fast passage principle causing transitions between particular magnetic substates. One strong field and weak field transition pair is located between the sextupoles and another pair between the second sextupole and the ionizer.

Each strong field transition consists of a magnetic field transverse to the beam direction (85 G for deuterons, $150 \mathrm{G}$ for protons) and an rf magnetic field (330 MHz, $400 \mathrm{MHz}$, $460 \mathrm{MHz}$ for deuterons and $1485 \mathrm{MHz}$ for protons) generated by a cavity. Each weak field transition consists of a magnetic field of $10 \mathrm{G}$ transverse to the beam direction and an rf magnetic field of $15 \mathrm{MHz}$ generated by a small coil with its axis along the beam. The strong field transition will be shielded from the fields of the sextupole magnet by a $1.6 \mathrm{~mm}$ thick iron field clamp. The weak field transitions are located inside a cylinder of magnetic shielding to prevent interference from other magnetic fields.

The mechanical design of the cavities follows the design of Robinson et al. ${ }^{1}$ For driving the cavities, we opted to change from a medium power signal generator to a selfoscillating loop utilizing the cavities self-resonance and a high gain amplifier. Assembly of the $330 \mathrm{MHz}$ and the $400-460 \mathrm{MHz}$ dual unit has been completed and tested for resonance, quality factor and vibration characteristics. Teflon shims have been inserted between the cavity elements to reduce vibration. The driving ports of the $330 \mathrm{MHz}$ and the $400 / 460 \mathrm{MHz}$ dual frequency cavities have been matched to the $50 \mathrm{Ohm}$ feeding cable impedance and power tests have been performed to determine thermal stability. After phase adjusting the output of the cavity with the input we were able to operate from 500 milliwatts to over 5 watts. Data taken thus far show we have approximately $10 \mathrm{G}$ per square root of $\mathrm{rf}$ power(W).

Both strong field rf transition magnets have been built and mapped. Difficulties with non-uniformity of field in the strong field magnets of the TUNL design have been solved by adding Rose shims to trim the field while keeping the pole tips short. The weak field transitions are currently being fabricated.

\section{ECR IONIZER}

The HIPIOS ECR ionizer design closely follows the TUNL design ${ }^{2}$ which has the hexapole magnet mounted within a vacuum chamber on which the external mirror solenoids and return yoke are assembled. We have, however, made several modifications to the basic configuration to address special requirements for our application, accommodate competing technical requirements, and incorporate improvements resulting from source commissioning work at TUNL.

A primary technical consideration involves the design and mounting of the permanent magnet ( $\mathrm{SmCo}$ ) hexapole, which can be damaged by plasma or beam heating above $300{ }^{\circ} \mathrm{C}$ 
or by exposure to the hydrogen gas in the ionizer vacuum chamber. An ECR chamber pressure less than $0.1 \mu$ Torr is required. A further complication is that the internal structure (hexapole magnet, pre-buncher, and extraction electrodes) of the ECR ionizer is isolated from and operated at $20 \mathrm{kV}$ above platform ground to provide an extraction gradient. This allows the majority of the ion source apparatus to remain at ground potential, and also simplifies the pre-bunching of the low energy dc beam from the ionizer for later injection into the IUCF cyclotrons.

To meet the competing requirements of vacuum, temperature, rf and high voltage constraints on the mounting of the hexapole structure, it was decided to vacuum encapsulate the permanent magnets in thin walled $(0.5 \mathrm{~mm})$ stainless steel cans welded onto thicker ring brackets to form the hexapole field configuration. The magnets are inserted into the welded structure following fabrication, mapped and shimmed to minimize other multipole field components, and then epoxy impregnated. This structure is supported from one end of the ECR vacuum chamber by six $2.5 \mathrm{~cm}$ diameter ceramic insulators. A separate freon cooled copper heat shield is mounted inside the hexapole structure to conduct the heat generated by the plasma and $\mathrm{rf}(\leq 150$ watts) away from the magnets. This overall design provides for an open geometry permitting good radial and axial pumping in the region of the ECR plasma.

The rf generator for the ECR plasma will operate over a range of 2.6 to $4.7 \mathrm{GHz}$ at 150 watts. The system will consist of a voltage controlled oscillator based on an Avantec HTO-2600 oscillator and AGT-8235 variable gain preamplifier. Final amplification of the microwave system is provided by a Keltec TWTA 200 watt amplifier. Power will be fed into the ECR chamber via Heliax coaxial cable to an internally mounted horn using a waveguide adapter at the chamber. We expect to achieve higher beam intensities and polarizations at the higher frequencies, as reported by the TUNL group.

Optimization of the ECR beam extraction, focusing and acceleration geometry for minimum beam phase space and maximum transmission of protons has been performed, with the invaluable help of T. Antaya, using the ray trace code BEAM3D specifically designed for ECR extraction at Michigan State University.

The ECR vacuum chamber and hexapole mounting structure have been fabricated, and we are currently assembling the hexapole for mapping. Fabrication of the remainder of the ECR assembly should be completed by the end of the month.

\section{BUNCHERS}

A prototype of the wideband, ramp-waveform buncher to be incorporated in HIPIOS was built and tested at low power by Lars Hermansson, a visiting engineer from The Swedberg Laboratory in Uppsala, Sweden. In order to compress $90 \%$ of the DC beam from the source into the $\pm 3^{\circ}$ cyclotron phase acceptance, this buncher should have a linearity of $\pm(30 / \mathrm{n}) \%$ of the voltage amplitude over $90 \%$ of the fundamental rf period, where $\mathrm{n}$ is the pulse-selection ratio $(\mathrm{n}=1,2,3)$ (i.e. $\mathrm{f}_{\text {buncher }}=\mathrm{f}_{\text {cyclotron }} / \mathrm{n}$ ). A buncher with this linearity specification should ideally produce a beam phase width of less than $\pm 30^{\circ}$ at the entrance to the resonant buncher in BL1C, which always operates at $f_{\text {cyclotron }}$, and the nonlinearity resulting from the sinusoidal waveform of this buncher should result in less than $\pm 1^{\circ}$ beam phase width at the cyclotron (neglecting all other effects). The test 
results are compared with the design specifications in Table 1. One can see that, for the most part, the specifications have been met or exceeded.

A mechanieal design for this buncher has been completed. The length of buncher has been increased from 0.25 to $0.35 \mathrm{~m}$ so that the buncher power amplifier rating can be reduced from 1000 to $500 \mathrm{~W}$. The electrical tests will be repeated after fabrication of the final unit.

The charge to mass ratio, $Q / A$, of beams from the HIPIOS will vary by a factor of at least four. For the BL1C resonant buncher, the buncher length which optimizes the transit time factor varies by more than a factor of $21 / 2$ as a function of $\mathrm{Q} / \mathrm{A}$ and cyclotron frequency. Consequently, a buncher optimized for beams with $Q / A=1$ would be totally ineffectual for beams with $Q / A=4$. A compromise buncher length would increase the amplifier power requirements by a factor of three. A second alternative would be to have two bunchers of different lengths. The third option, which we are currently exploring, is to have a single buncher with a variable length drift electrode.

Table I

Linearity Measurements as a function of frequency.

\begin{tabular}{|c|c|c|c|c|}
\hline$n$ & \multicolumn{2}{|c|}{$\begin{array}{c}\text { Linearity } \\
( \pm \%)\end{array}$} & $\begin{array}{c}\text { Fractions of Period } \\
(\%)\end{array}$ \\
\hline & Specification & Achieved & Specification & Achieved \\
\hline 1 & 5 & 5 & 90 & 91 \\
\hline 2 & 10 & 5 & 90 & 88 \\
\hline 3 & 15 & 10 & 90 & 81 \\
\hline
\end{tabular}

\section{TERMINAL C BEAM TRANSPORT}

Design of the optics for transporting the beam from the source to the acceleration column has been completed (Fig. 3). The magnetic steerers have been assembled and are awaiting mapping. A prototype einzel lens has been installed in Terminal A for testing, and a prototype slit assembly is currently being fabricated. Designs for the electrostatic channel, vertical translator magnets and spin rotation solenoid will be completed over the summer.

\section{TERMINAL C}

Detailed design work awaits the availability of the mechanical design staff, but a number of studies and tests are in progress. These include high voltage testing of a commercial ceramic support leg and a G-10 insulated drive shaft for AC power, research on a suitable drive motor for the insulated drive shaft, determination of filter needs for 


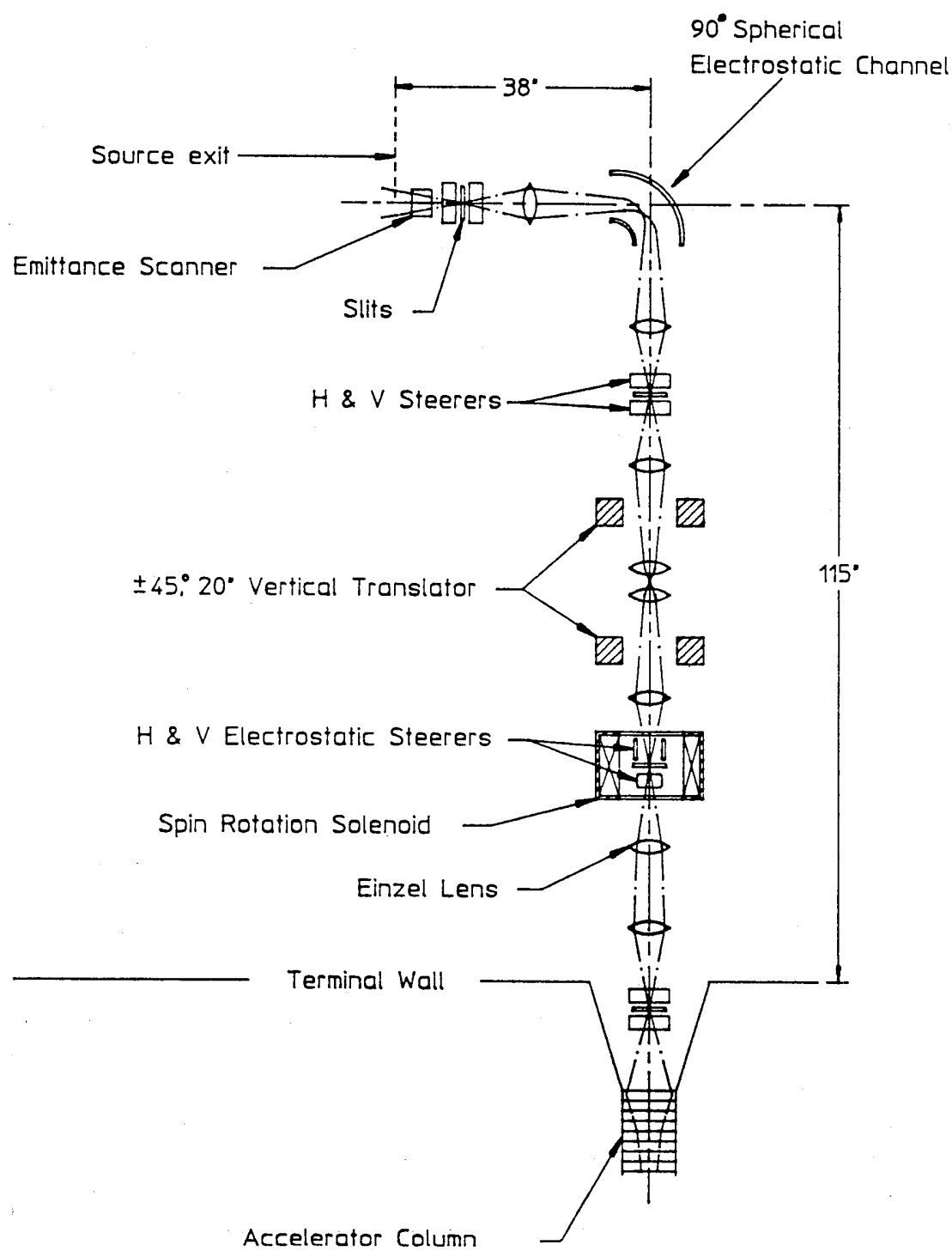

Figure 3. Terminal C Beam Transport

the $\mathrm{AC}$ power lines and design of new voltage-graded water lines for supplying de-ionized water to the terminal through the $600 \mathrm{kV}$ potential.

VII. BEAM LINE 1C

The BL1C design can be divided into two principal parts: the bunching and transfer of beam from the terminal to the cyclotron and matching the beam from the terminal to the cyclotron.

The beam line is composed of $180^{\circ}$ betatron phase advance sections between each $45^{\circ}$ dipole. This design automatically leads to alternating sections of high and vanishing dispersion. The high energy resonant buncher will go in one of the two zero dispersion 
sections. This symmetry also suggests a natural ordering of the steering dipoles and beam position measurement system: both steering magnets and position monitors are located modula $90^{\circ}$ in betatron phase advance. This placement makes both local beam movement and steering simpler for the operator. Although we plan to have the computer tune the beam line using feedback from the beam diagnostic systems, we will also incorporate automatic display of the position monitor $90^{\circ}$ downstream if the operator selects an individual steerer.

In addition to beam position monitors, there are wire scanners allowing measurement of the beam emittance at the beginning of the line so that the first four quadrupoles can be properly set, as well as another emittance measurement system downstream to check that the matching has been properly done. Radio frequency nonintercepting pickups are extensively employed. Besides being used to measure the beam position, various pickups will be used to provide automatic phase feedback to both the terminal buncher and high energy buncher. In addition, high bandwidth longitudinal pickups are being developed to allow realtime viewing of the beam bunching (i.e., viewing of the longitudinal focussing of the beam).

Design of the beam line optics using TRANSPORT has progressed far enough to establish many of the basic features of the line, including the number of quadrupole magnets (21) and the number of magnetic steerers (22). The dipole magnet count remains at six. Magnetic designs of the BL1C dipole and quadrupole magnets are complete, including preliminary coil designs and a general description of the power supplies required.

In order to complete the optics calculations, we mapped the six dimensional shape of the cyclotron system acceptance at various points. This will allow us to better match the beams from the terminals to the injector. Attention is now focussed on beam bunching and the diagnostics described above, as well as developing a potential pencil beam scanning system for optimizing beam transmission to the cyclotron.

\section{CONTROL SYSTEM}

We decided not to use the existing cyclotron control systems for HIPIOS since their limited functionality and numerous obsolete components placed limits on the proposed expansion. Instead, we are implementing a new system that will operate in parallel with the present controls and provide a platform for future facility expansion. A detailed description is included elsewhere in this report.

1. H. G. Robinson, et al., Nucl. Instrum. Methods, A278, 655 (1989).

2. T. B. Clegg, U. Konig, P. A. Schmelzbach and W. Grüebler, Nucl. Instrum. Methods, A238, 195 (1985). 